\title{
Genes for degradation and utilization of uronic acid- containing polysaccharides of a marine bacterium Catenovulum sp. CCB-QB4
}

\author{
Go Furusawa $^{\text {Corresp., } 1 \text {, Nor Azura Azami }}{ }^{1}$, Aik-Hong Teh ${ }^{1}$ \\ ${ }^{1}$ Centre for Chemical Biology, Universiti Sains Malaysia, Bayan Lepas, Penang, Malaysia \\ Corresponding Author: Go Furusawa \\ Email address: furusawa@usm.my
}

\section{Background}

Oligosaccharides from polysaccharides containing uronic acids are known to have many useful bioactivities. Thus, polysaccharide lyases (PLs) and glycoside hydrolases (GHs) involved in producing the oligosaccharides have attracted interest in both medical and industrial settings. The numerous polysaccharide lyases and glycoside hydrolases involved in producing the oligosaccharides were isolated from soil and marine microorganisms. Our previous report demonstrated that an agar-degrading bacterium, Catenovulum sp. CCB-QB4, isolated from a coastal area of Penang, Malaysia, possessed 183 glycoside hydrolases and 43 polysaccharide lyases in the genome. We expected that the strain might be degraded and use uronic acid-containing polysaccharides as a carbon source, indicating that the strain has a potential for a source of novel genes for degrading the polysaccharides.

\section{Methods}

To confirm the expectation, the QB4 cells were cultured in artificial seawater media with uronic acidcontaining polysaccharides, namely alginate, pectin (and saturated galacturonate), ulvan, and gellan gum, and the growth was observed. The genes involved in degradation and utilization of uronic acidcontaining polysaccharides were explored in the QB4 genome using CAZy analysis and BlastP analysis.

\section{Results}

The QB4 cells were capable of using these polysaccharides as a carbon source, and especially, the cells exhibited a robust growth in the presence of alginate. $28 \mathrm{PLs}$ and $22 \mathrm{GHs}$ related to degradation of these polysaccharides were found in the QB4 genome based on the CAZy database. 11 polysaccharide lyases and 16 glycoside hydrolases contained lipobox motif, indicating that these enzymes play an important role in degrading the polysaccharides. 14 of 28 polysaccharide lyases were classified into ulvan lyase, and the QB4 genome possessed the most abundant ulvan lyase genes in the CAZy database. Besides, genes involved in uronic acid metabolisms were also present in the genome. These results were consistent with cell growth. In the pectin metabolic pathway, the strain had genes for three different pathways. However, the growth experiment using saturated galacturonate exhibited that the strain can only use the pathway related to unsaturated galacturonate. 
1 Genes for degradation and utilization of uronic acid-containing polysaccharides of a

2 marine bacterium Catenovulum sp. CCB-QB4

3

4 Go Furusawa* Nor Azula Binti Azami, and Aik-Hong Teh

5

6 Author's institutional affiliation:

7 Centre for Chemical Biology, Universiti Sains Malaysia, 10 Persiaran Bukit Jambul, 11900

8 Bayan Lepas, Penang, Malaysia

9

10 Contact information;

11 Go Furusawa

12 Centre for Chemical Biology, Universiti Sains Malaysia, 10 Persiaran Bukit Jambul, 11900

13 Bayan Lepas, Penang, Malaysia

14 E-mail: furusawa@usm.edu.my

15

16

17

18

19

20

21

22

23

24

25

26

27

28

29

30

31

Peer) reviewing PDF | (2020:06:50285:3:0:NEW 31 Dec 2020) 


\section{Abstract \\ 33 Background}

34 Oligosaccharides from polysaccharides containing uronic acids are known to have many useful 35 bioactivities. Thus, polysaccharide lyases (PLs) and glycoside hydrolases (GHs) involved in 36 producing the oligosaccharides have attracted interest in both medical and industrial settings.

37 The numerous polysaccharide lyases and glycoside hydrolases involved in producing the 38 oligosaccharides were isolated from soil and marine microorganisms. Our previous report 39 demonstrated that an agar-degrading bacterium, Catenovulum sp. CCB-QB4, isolated from a 40 coastal area of Penang, Malaysia, possessed 183 glycoside hydrolases and 43 polysaccharide 41 lyases in the genome. We expected that the strain might be degraded and use uronic acid42 containing polysaccharides as a carbon source, indicating that the strain has a potential for a 43 source of novel genes for degrading the polysaccharides.

\section{Methods}

45 To confirm the expectation, the QB4 cells were cultured in artificial seawater media with uronic 46 acid-containing polysaccharides, namely alginate, pectin (and saturated galacturonate), ulvan, 47 and gellan gum, and the growth was observed. The genes involved in degradation and utilization 48 of uronic acid-containing polysaccharides were explored in the QB4 genome using CAZy 49 analysis and BlastP analysis.

\section{Results}

51 The QB4 cells were capable of using these polysaccharides as a carbon source, and especially, 52 the cells exhibited a robust growth in the presence of alginate. 28 PLs and 22 GHs related to 53 degradation of these polysaccharides were found in the QB4 genome based on the CAZy 54 database. 11 polysaccharide lyases and 16 glycoside hydrolases contained lipobox motif, 55 indicating that these enzymes play an important role in degrading the polysaccharides. 14 of 28 56 polysaccharide lyases were classified into ulvan lyase, and the QB4 genome possessed the most 57 abundant ulvan lyase genes in the CAZy database. Besides, genes involved in uronic acid 58 metabolisms were also present in the genome. These results were consistent with cell growth. In 59 the pectin metabolic pathway, the strain had genes for three different pathways. However, the 60 growth experiment using saturated galacturonate exhibited that the strain can only use the 61 pathway related to unsaturated galacturonate. 


\section{Introduction}

64 Uronic acids are a class of sugar acids oxidized the hydroxyl group on C6 of aldoses. Uronic acids including D-glucuronic acid, D-galacturonic acid, D-mannuronic acid, L-guluronic acid, and Liduronic acid are components of polysaccharides produced by animals (heparin), terrestrial plants

67 (pectin), seaweed (alginate and ulvan), and bacteria (gellan gum) (de Lederkremer and Marino, 2003). In general, these polysaccharides such as pectin, alginate, ulvan, and gellan gum, have broad potential in many applications due to their excellent properties of biocompatibility, non-toxic, immunogenicity, availability, and relatively low cost (Morelli. A. and Chiellini, 2010; Venkatesan et al., 2015; Rahman et al., 2017).

Pectin is a polymer with a linear structure characterized by a backbone consisting of a few hundred to thousand D-galacturonic acid units linked together by $\alpha-(1 \rightarrow 4)$-glycosidic linkages. It found in the cell walls of the plant and intracellular layer of plant cells, mainly fruits, such as apples, oranges, and lemons (Mudgil, 2017). Pectin contains a significant amount of neutral sugar, typically L-rhamnose, L-arabinose, D-galactose, D-xylose, and D-glucose which linked to the hydroxyl groups on the number 2 and 3 carbons of the main chain. Pectin attracted attention due to its gelling capabilities (Penhasi and Meidan, 2015). Pectin is widely used as a thickener and stabilizing agent in the food industry (Munarin et al., 2012).

Alginate, also known as alginic acid is an unbranched polymer composed of $\beta$-Dmannuronic acid (M) and $\alpha$-L-guluronic acid (G), which are covalently (1-4)-liked. The residues are randomly arranged into MM-, GG- and MG-blocks (Meng and Liu, 2013). Alginate is distributed widely in the cell wall of marine brown agar and has long been used in the industry such as medical field, fabric, food, and beverage industries as thickening, gel-forming, and colloidal stabilizing agents (Liakos et al., 2013; Martău et al., 2019).

Ulvan is water-soluble polysaccharides found in the cell wall of green algae (Ulva and Enteromorpha) composed mainly of 3-sulfated rhamnose (Rha3S), glucuronic acid (GlcA), iduronic acid (IdoA), and xylose (Xyl) (Kim et al., 2011). Ulvan has attracted pharmaceutical and medical applications for its anti-viral, anti-coagulant, and anti-proliferative activities towards cancer cells and its immune-stimulating properties (Alves, Sousa \& Reis, 2013). In addition, ulvan also showed to be an activator of plant defense and an inducer of plant resistance (Alves, Sousa \& Reis, 2013). Therefore, it is a good potential for agricultural applications. 
93

94 95

96

97

98

99

100

101

102

103

104

105

106

107

108

109

110

111

112

113

114

115

116

117

118

119

120

121

122

123

Gellan gum is an exopolysaccharide produced from non-pathogenic, Gram-negative bacterium, Sphingomonas elodea (earlier Pseudomonas elodea) using aerobic fermentation (Vendrusculo et al., 1994). Gellan gum consisting of repeating tetrasaccharide units of glucose, glucuronic acid, and rhamnose residues in a 2:1:1 ratio: $[\rightarrow 3)-\beta$-D-glucose-( $1 \rightarrow 4)-\beta$-D-glucuronic acid-( $1 \rightarrow 4)-\beta$-D-glucose- $(1 \rightarrow 4)$ - $\alpha$-L-rhamnose- $(1 \rightarrow]$ (Jansson et al., 1983). Gellan gum has been used in medicine, pharmaceutical formulations, cosmetics, or tissue engineering. As a biocompatibility polysaccharide, gellan gum is used in contact with or inside the body. Besides, gellan gum also useful in the food and biotechnology industry as immobilization of enzymes and yeast cells (Iurciuc (Tincu) et al., 2015).

These polysaccharides could also be alternative sustainable sources for fermentative biofuel production (John et al., 2011). The PLs and GHs play an important role in the saccharification of the polysaccharides in the process of biofuel production (Edwards et al., 2011; Takeda et al., 2011; Li et al., 2015). For example, endo- and exolytic alginate lyases from Saccharophagus degradans A1 were co-displayed on the yeast cell surface, and the co-displaying yeasts were able to effectively produce monosaccharides (Takagi et al., 2016b). On the other hand, oligosaccharides from uronic acids generated by PLs are known to have multiple biological activities. For instance, alginate oligosaccharides stimulate the growth of human endothelial (Kawada et al., 1997) and keratinocytes cells (Kawada et al., 1999). In addition, the oligosaccharides also promote the growth and root elongation of rice and barley (Tomoda et al., 1994; Hien et al., 2000). Mandalari and colleagues reported that pectin oligosaccharides (POS) have probiotic effects through the improvement of bifidobacteria and lactobacillus (Mandalari et al., 2007). Besides, POS inhibited inflammation, fibrosis formation, as well as cancer progression, transformation, and metastasis (Bonnin Garnier \& Ralet, 2014). Thus, PLs and GHs have attracted considerable interest in both academic and commercial spheres.

Many bacterial species, such as the genera Agrobacterium, Bacillus, Cellulophaga, Clostridium, Erwinia, Flammeovirga, Flavobacterium, Microbulbifer, Psuedoalteromonas, Pseudomonas, Saccharophagus, Sphingomonas, Vibrio, Xanthomonas, and Zobellia, are known as bacteria that are capable of degrading uronic acid-containing polysaccharides, mainly alginate and pectin (Liu et al., 2019; Dubey et al., 2016; Takagi et al., 2016). The genus Catenovulum consisted of three species, C. agarivorans, $C$. maritimum, and $C$. sediminis, was also known to degrade agar (all three strains) and alginate (C. maritimum, and C. sediminis). Besides that, 
124 Catenovulum sp. LP was able to produce an ulvan lyase (Li et al., 2015; Qiao et al., 2020).

125 However, the degradation and utilization pathway of uronic acid-containing polysaccharides

126 containing alginate, gellan gum, pectin, and ulvan by the genus Catenovulum are poorly

127 understood. Our group isolated Catenovulum sp. CCB-QB4 (referred to hereafter as QB4) from

128 Queens Bay of Penang, Malaysia, and the complete genome sequence was reported (Lau et al., 129 2019). From the study, it was reported that the QB4 genome contained 183 GHs and 43 PLs. Based 130 on the information, this bacterium is predicted to have the ability to utilize many polysaccharides.

131 To confirm the expectation, in this study, the cell growth of QB4 in the presence of polysaccharides 132 containing uronic acids, namely, alginate, pectin, ulvan, and gellan gum, was confirmed using a 133 shake flask fermentation method. In addition, genes involved in degrading and utilizing these 134 polysaccharides were explored in the QB4 genome. This study is the first report to describe the 135 degradation and probable metabolic pathways for utilization of four different uronic acid136 containing polysaccharides in the genus Catenovulum.

137

138 Materials and Methods

139

\section{Strain and chemicals}

141 The QB4 cells (Lau et al., 2019) was cultured using high nutrient artificial seawater medium (H142 ASWM) condition $[0.5 \%$ tryptone, 2.4\% (w/v) artificial sea salt mix (Marine Enterprises 143 International), 10mM HEPES, pH 7.6], as reported by Furusawa and co-workers, 2015 (Furusawa 144 et al., 2015). $0.1 \%$ of polygalacturonic acid [pectic acid] (Nacalai Tesque), sodium alginate 145 (Sigma-Aldrich), Gelzan ${ }^{\mathrm{TM}}$ CM (gellan gum) (Sigma-Aldrich), and ulvan were used as carbon 146 source throughout the research.

\section{Purification of ulvan}

149 The ulvan was purified according to the method described by Tabarsa et al. (2012) with slight 150 modification. $5 \mathrm{~g}$ of the dry powder of Ulva pertusa was dissolved and stirred in $100 \mathrm{~mL}$ of water 151 and stirred at $65^{\circ} \mathrm{C}$ for $3 \mathrm{~h}$. The mixture was cooled and centrifuged at $10,000 \mathrm{~g}$ for $20 \mathrm{~min}$ at 15 $152{ }^{\circ} \mathrm{C}$ using Sorvall ${ }^{\mathrm{TM}} \mathrm{RC} 6$ Plus Centrifuge (ThermoFisher Scientific). 4 volume of cold isopropanol 153 was added into the supernatant to precipitate crude polysaccharide. The solution was left at $4{ }^{\circ} \mathrm{C}$ 
154 for overnight. The crude ulvan was harvested by filtration, washed with $70 \%$ isopropanol several 155 times, and dried overnight at $60^{\circ} \mathrm{C}$.

156

\section{Determination of bacterial growth}

158 The QB4 cells were inoculated in $10 \mathrm{~mL}$ of H-ASWM broth and cultured overnight at $30^{\circ} \mathrm{C} .0 .1$ $159 \mathrm{~mL}$ of the cell suspension was inoculated into $100 \mathrm{~mL}$ of $\mathrm{H}-\mathrm{ASWM}$ medium with $0.2 \%$ of each 160 uronic acid as the carbon sources. Media with and without $0.2 \%$ glucose (Fisher Scientific) were 161 used as positive and negative controls, respectively.

162 Each flask was inoculated with $0.1 \mathrm{~mL}$ of pre-cultured bacterial cell suspension and incubated at $16330{ }^{\circ} \mathrm{C}$ with an agitation speed of $200 \mathrm{rpm}$ on the orbital shaker. To monitor the bacterial growth, 164 the colony-forming unit (CFU) counts were conducted by culturing the cells on H-ASWM agar 165 plates because the optical density measurement was not suitable for the sample with gelzan in

166

167 168

169

170

171

172

173

174

175

176

177

178

179

180

181

182

184

183 The complete genome of QB4 deposited at GenBank under the accession number CP026604which the broth was solidified by calcium ion contained in H-ASWM medium. $100 \mu$ of the cell suspension from each sample was collected every 3 hours. The suspension was diluted into $900 \mu 1$ of H-ASWM medium. After that, sequential 10-fold serial dilutions were made, and $100 \mu 1$ of aliquots of each dilution were plated on H-ASWM agar plates. Colonies were scored after incubation for $48 \mathrm{~h}$ at $30^{\circ} \mathrm{C}$, and the growth curve was constructed. The experiment was performed in triplicates. The generation time $(\mathrm{G})$ was estimated by the method described by Aparna et al. based on the growth curve (Aparna et al., 2020). The number of generation (n) was determined by the method of $n=(\log b-\log B) / \log 2$, where $B$ and $b$ are the number of bacteria at the beginning of a time interval and at the end of the time interval, respectively. The generation time $(\mathrm{G})$ was determined by the method of $\mathrm{G}=\mathrm{t} / \mathrm{n}$, where $\mathrm{t}$ is the time interval ( $\mathrm{min}$ ).

To confirm the utilization of saturated galacturonate, the QB4 cells were culture in an H-ASWM medium with $0.2 \%$ saturated galacturonate. Media with and without $0.2 \%$ glucose was used as positive and negative controls, respectively. To monitor the bacterial growth, the optical density $\left(\mathrm{OD}_{600 \mathrm{~nm}}\right)$ was measured at $3 \mathrm{~h}$ intervals for $30 \mathrm{~h}$ using UV spectrophotometer UV-1800 (Shimadzu). The experiment was performed in triplicates.

\section{Genomic analysis of Catenovulum sp. CCB-QB4} CP026605 was determined by Lau et al. (Lau et al., 2019). Genes involved in the carbohydrate- 
185 active enzyme (CAZymes) in QB4 were predicted using dbCAN pipelines (Yin et al., 2012). 186 Several enzymes related to uronic acid metabolism in QB4 were predicted by Blastp at the National 187 Center for Biotechnology Information (NCBI) server (Bethesda, MD, U.S.A.) and were found 188 using the Kyoto Encyclopedia of Genes and Genomes (KEGG) pathway database (Kanehisa and 189 Goto, 2000). Finally, the amino acid sequence similarity of all enzymes was confirmed by using 190 Blastp analysis with Protein Data Base (PDB). Signal peptide prediction was conducted by LipoP 1911.0 (Juncker et al., 2003) and SignalP 5.0 (Armenteros, et al., 2017). For sequence alignment of 192 DEH reductase, the amino acid sequence of A1-R and A1-R' from Sphingomonas sp. A1 and DEH 193 reductase from Saccharophagus degradans 2-40 were obtained from GenBank 194 (https:/www.ncbi.nlm.nih.gov/genbank/). The sequence alignment with 4 sequences was 195 conducted by ClustalW (Thompson et al., 1994) at PRABI Lyon-Gerland (https://npsa196 prabi.ibcp.fr/cgi-bin/npsa_automat.pl?page=/NPSA/npsa_clustalw.html).

197

198

199

Results

200 Growth confirmation of QB4 using uronic acids.

201 To confirm the growth of QB4 in the presence of uronic acids, the cells of QB4 were cultured in 202 H-ASWM with alginate, pectin, ulvan, and gelzan. As shown in figure 1, the cells of QB4 203 showed good growth in the presence of the uronic acid-containing polysaccharides and glucose 204 except for negative control. The cells of QB4 reached the early stationary phase at $9 \mathrm{~h}$ in $\mathrm{H}-$ 205 ASWM with alginate and gelzan. The generation time of alginate and gelzan was 76.76 and $20674.07 \mathrm{~min}$. However, the cell number of the sample with gelzan was 5.7 times lower than that of 207 the sample with alginate. On the other hand, the cells cultured by pectin and glucose reached the 208 early stationary phase at $18 \mathrm{~h}$. Besides that, in the case of using ulvan, the cells reached 209 stationary phase at $24 \mathrm{~h}$, and the sample exhibited the highest cell number at $30 \mathrm{~h}$ in the 210 experiment. The generation time of the samples with glucose, pectin, and ulvan was 91.37, 21195.74 , and $85.10 \mathrm{~min}$, which were longer than that of the samples with alginate and gelzan. Thus, 212 QB4 cells exhibited a robust growth in the presence of uronic acids. Subsequently, we focused 213 on genes for degradation and utilization of uronic acids in the genome of QB4.

\section{Genes for alginate degradation and utilization}


216 Alginate is a major polysaccharide found in the cell wall of brown algae and consisted of 217 guluronate and mannuronate arranged as 1,4-linked polysaccharides. First, alginate is degraded 218 into oligomeric or monomeric units by alginate lyases. Eight alginate lyase genes were found in 219 the QB4 genome (Table 1). Ad1-PL6, Ad2-PL6 and Ad3-PL6, Ad4-PL7, Ad5-PL7, Ad6-PL7, 220 Ad7-PL7, and Ad8-PL17 were classified into family PL6, PL7, and PL17 based on the CAZy 221 database, respectively. As a result of Blastp search with Protein Data Bank (PDB), Ad proteins 222 were similar to Alyge from Paraglaciecola chathamensis (Ad1-PL6 and Ad3-PL6), AlyQ from 223 Persicobacter sp. CCB-QB2 (Ad2-PL6, Ad4-PL7, and Ad5-PL7), alginate lyase of Klebsiella 224 pneumoniae (Ad6-PL7 and Ad7-PL7) and Saccharophagus degradans 2-40 (Ad8-PL17) (Table S1). LipoP 1.0 (Juncker et al., 2003) was used to predict the signal peptides and their type of each enzyme. Table 1 demonstrated that Ad1-PL6, Ad2-PL6, Ad4-PL7and Ad5-PL7 possessed type I signal peptide, which was cleaved by signal peptidase I, and Ad6-PL7, Ad7-PL7 and Ad8PL17 have type II lipoprotein signal peptide which was cleaved by signal peptidase II. The lipoprotein signal peptide referred to as "lipobox" plays an important role in anchoring the protein on the outer surface of the cell membrane after secretion and modification of N-terminal cysteine residue (Pugsley et al., 1986; Hutcheson et al., 2011). This suggested that Ad6-PL7, Ad7-PL7, and Ad8-PL17 may localize on the cell surface. In contrast, Ad1-PL6, Ad2-PL6, Ad4PL7, and Ad5-PL7 may be released into the culture medium. On the other hand, Ad3-PL6, which does not possess any signal peptides, may locate at the cytoplasm.

Takase et al. (Takase et al., 2010) described that an exotype oligoalginate lyase, A1-IV, from Sphingomonas sp. A1 degraded oligoalginates into monosaccharides, which are then nonenzymatically converted to 4-deoxy-L-erythro-5-hexoseulose uronic acid (DEH). Takagi and colleagues also reported that an alginate lyase, Alg7K, from Saccharophagus degradans showed exolytic activity and produced monosaccharides from oligoalginates (Takagi et al., 2016a). The amino acid sequence of the alginate lyase, Ad6-PL7, showed high similarity $(77.3 \%)$ to that of Alg7K, suggesting that Ad6-PL7 might mediate hydrolysis of oligoalginate to produce monomers. The DEH is converted to 2-keto-3-deoxy-D-gluconate (KDG) by NADH or NADPHdependent DEH reductase (Takase et al., 2010; Takase et al., 2014; Kim et al., 2016). A result of the BLASTp search demonstrated that the SDR family oxidoreductase, Au1, found in the QB4 genome (Table 1) showed high similarity to NADH (A1-R', $60.9 \%$ ) and NADPH (A1-R, 50.8 \%)-dependent DFH reductase. In addition, the gene aliments of DEH reductases showed that the 
247 TGXXXGX motif and catalytic triad (Ser, Tyr, and Lys) are highly conserved in SDR family

248 enzymes (Takase et al., 2014) were conserved in the SDR family oxidoreductase (Fig. S1). These

249 results indicated that Au1 might function as DEH reductase. KDG kinase catalyzes the

250 conversion of KDG to 2-keto-3-deoxy-phosphogluconate (KDPG), and then KDGP is converted

251 into D-glyceraldehyde-3-phosphate and pyruvate by 2-dehydro-3-deoxy-phosphogluconate

252 aldolase via Entner -Doudoroff pathway. Two of the genes encoding the KDG kinase, Au2 and

$253 \mathrm{Au} 3$, and the aldolase, Au4, and Au5, were found in the genome of QB4 (Table 1). Finally,

254 pyruvate produced by the pathway goes into further metabolic pathways for generating energy.

255 The five Au proteins also demonstrated a high degree of amino acid sequence similarity $(>50 \%)$

256 to DEH reductase A1-R' from Sphingomonas sp. A1 (Au1), KDG kinase Shigella flexneri (Au2

257 and 3), 2-keto-3-deoxy-6-phosphogluconate aldolase from Thermotoga maritima (Au4), and

258 Kdpg aldolase from Escherichia coli (Au5) (Table S1). As mentioned above, QB4 possessed

259 many alginate lyases and enzymes responsible for alginate utilization. This is consistent with

260 robust growth of QB4 in the presence of alginate, as shown in Fig. 1.

261

\section{Genes for pectin degradation and utilization}

263 As a first step, pectin is depolymerized by pectin lyases or polygalacturonases. Pectin and pectate

264 lyases are classified into five families of PLs. Although pectin lyases attack highly methyl-

265 esterified pectin, pectate lyases specifically attack non-methylated polygalacturonate or

266 methylated pectin with a very low degree (Hugouvieux-Cotte-Pattat et al., 2014). These enzymes

267 degrade pectin to unsaturated pectic-oligosaccharides and disaccharides.

268 In the CAZy database, seven pectate lyases belonging to family PL1 (Pd1_PL1, Pd2_PL1,

269 Pd3_PL1, Pd4_PL1, and Pd5_PL1), PL3 (Pd6_PL3), and PL10 (Pd7_PL10) family pectate

270 lyases were found in the genome of QB4. The result of Blastp search using PDB database on Pd

271 proteins also demonstrated that these Pd proteins were similar (>45\%) to pectate lyase

272 (Pd1_PL1, Pd2_PL1, Pd3_PL1, Pd5_PL1, Pd6_PL3, and Pd7_PL10) and pectinesterase

273 (Pd4_PL1) from other bacterial species (Table S2). CBM13 found in Pd1_PL1 and Pd6_PL3

274 and CBM35 found in Pd2_PL1 and Pd3_PL1 were capable of binding to multi-ligands, such as

275 arabinan, arabinoxylan, and pectin (Fujimoto, 2013; Dhillon et al., 2018). However, CBM77

276 contained in Pd2_PL1 recognized homogalacturonan (Fujimoto 2013). Pectin methylesterase

277 (PME) domain from family 8 Carbohydrate Esterase (CE8) was found in Pd4_PL1 and 
278 Pd5_PL1. PMEs produce de-esterified homogalacturonan by catalyzing the de-esterification of

279 the methoxyl group of the pectin; as a result, the products are effectively degraded by pectate 280 lyases (Kashyap et al., 2001). Besides, although Pd1_PL1, Pd3_PL1, Pd4_PL1, and Pd7_PL10

281 possessed type I signal peptide, Pd5_PL1 and Pd6_PL3 had lipobox, indicating that both two

282 lyases localize on the cell surface. Hence, these results suggested that Pd5_PL1 plays an

283 important role in the pectin degradation of QB4 due to its de-esterification and depolymerization

284 activities and its localization.

285 The unsaturated disaccharides generated by the pectate lyases are converted to 5-keto-4286 deoxyuronate (DKI) by two distinct processes. One is that the disaccharides are degraded by 287 oligogalacturonate lyase belonging to family PL22, and then the product, $\Delta-4,5$-unsaturated 288 galacturonate, is linearized into DKI by KdgF (Hobbs et al., 2016). The other is that unsaturated 289 290 galacturonyl hydrolases belonging to GH105 degrades the disaccharides and directly releases

291

292

293

294

295

296

297

298

299

300

301

302

303

304

305

306

307

308

DKI (Hobbs et al., 2019). In the QB4 genome, no PL22 enzymes were found in the genome.

However, three GH105 proteins, Pd8_GH105, Pd9_GH105, and Pd10_GH105, were present in the genome (Table 2). These enzymes showed a high degree of similarity (49 $67 \%$ ) on unsaturated rhamnogalacturonyl hydrolases (YteR) based on Blastp search with PDB database.

These results indicated that oligogalacturonate might directly convert to DKI by GH105 proteins in QB4. DKI is converted to 2-keto-3-deoxygluconate (KDG) by two enzymes, a DKI isomerase (KduI) and a 2-dehydro-3-deoxy-D-gluconate 5-dehydrogenase (KduD) (Hobbs et al., 2019). The pathway analysis based on KEGG showed that four of $\mathrm{KduI}(\mathrm{Pu} 1, \mathrm{Pu} 2, \mathrm{Pu} 3$, and $\mathrm{Pu} 4)$ and $\mathrm{KduD}$ (Pu5, Pu6, Pu7, and Pu8) were present in the genome of QB4. Blastp search with PDB database also exhibited that these enzymes showed a high degree of similarity (63 $71 \%)$ on KduD and KduI from Enterococcus faecalis, Escherichia coli, and a pectolytic bacterium, Pectobacterium carotovorum (Table S2). These results indicated that QB4 use pectin as a carbon source for cell growth and is consistent with the result shown in Fig. 1.

On the other hand, the pathway analysis also showed the other pathway using saturated galacturonate (monosaccharide) produced by polygalacturonases. As mentioned above, although QB4 possesses seven pectate lyases, only two polygalacturonases (GH28), Pd11_GH28 and Pd12_GH28, which were highly similar (>66\%) to polygalacturonase from pectolytic bacteria, such as Erwinia carotovora and Thermotoga maritima (Table S2), were found in the genome. Based on SignalP 5.0 analysis, it was found that Pd11_GH28 and Pd12_GH28 contained Tat and 
309 type I signal peptide, respectively. Saturated galacturonate is converted to D_tagaturonate by

310 glucuronate isomerase. Three glucuronate isomerases, $\mathrm{Pu}$ 9, $\mathrm{Pu}$ 10, and $\mathrm{Pu} 11$, which were highly

311 similar (>\%) to glucuronate isomerase of Salmonella enterica subsp. enterica serovar

312 Typhimurium and uronate isomerase of Caulobacter vibrioides CB15 (Table S2), were found in

313 the QB4 genome. The conversion of D.tagaturonate to KDG occurs through two distinctly

314 different pathways. One is that the process consists of two steps involving tagaturonate reductase

315 and altronate hydrolase and one intermediate, D-altronate (Richard and Hilditch, 2009). The

316 other is that tagaturonate is converted via three steps involving tagaturonate epimerase,

317 fructuronate reductase, and mannonate dehydratase and two intermediates, D-fructuronate and D-

318 mannonate (Valk et al., 2020). As shown in Table 2, QB4 possessed all genes involved in the

319 two different pathways, namely tagaturonate reductase (Pu12), altronate hydrolase (Pu13),

320 tagaturonate epimerase (Pu14), fructuronate reductase (Pu15), and mannoate dehydratase (Pu16).

321 Homologous enzymes of these proteins were also found in the PDB database (Table S2). This

322 result suggested that QB4 is also capable of using saturated galacturonate as a carbon source. To

323 confirm which pathway is functioning when QB4 cells grew in H-ASWM broth with

324 polygalacturonic acid, the QB4 cells were cultured in H-ASWM broth with polygalacturonic

325 acid or saturated galacturonate. As shown in Figure 2, although the cells with glucose

326 demonstrated robust growth, the cells with were unable to grow in the broth with saturated

327 galacturonate as well as the negative control in the incubation period. This result indicated that

328 the unsaturated galacturonate utilization pathway is the main pathway to utilize polygalacturonic

329 acid of QB4. Interestingly, genes for all pectate lyases, polygalacturonases, and one set of KduI

$330(\mathrm{Pu} 4), \mathrm{KduD}(\mathrm{Pu} 8)$, glucuronate isomerase $(\mathrm{Pu} 11)$, and tegaturonate reductase were located in a

331 plasmid (https://www.ncbi.nlm.nih.gov/nuccore/NZ_CP026605.1) found in QB4 (Table 2).

332

333 Genes for ulvan degradation and utilization

334 Ulvan lyases classified into 5 families, PL24, PL25, PL28, PL 37, and PL40 in the CAZy

335 database (Li et al., 2020) were isolated from several marine bacteria, such as genera,

336 Alteromonas, Pseudoalteromonas, Formosa, and Nonlabens (Kopel et al., 2016; Qin et al., 2018;

337 Ulaganathan et al., 2018a; Ulaganathan et al., 2018b; Reisky et al., 2019). Ulvan lyases cleave

338 between L-rhamnose 3-sulfate (Rha3S) and D-glucuronic acid (GlcA) or L-iduronic acid (IdoA).

339 Fourteen ulvan lyases in QB4 were classified into three PLs families, PL24 (Ud1_PL24, 
340

341

342

343

344

345

346

347

348

349

350

351

352

353

354

355

356

357

358

359

360

361

362

363

364

365

366

367

368

369

370

Ud2_PL24, Ud3_PL24, Ud4_PL24, Ud5_PL24, Ud6_PL24, Ud7_PL24, Ud8_PL24, and Ud9_PL24), PL25 (Ud10_PL25, Ud11_PL25, Ud12_PL25, and Ud13_PL25) and PL40

(Ud14_PL40). Ud proteins belonging to the PL24 family were highly similar ( $>62 \%$ ) to short ulvan lyase of Alteromonas sp. LOR, and Ud proteins belonging to the PL25 family were highly similar ( $>57 \%$ ) to ulvan lyase-PL25 of Pseudoalteromonas sp. PLSV (Table S3). The number of the ulvan lyases was obviously higher than that of $F$. agariphila (three PL40 proteins) (Reisky et al., 2019) and Alteromonas sp. LOR (one PL24 and one nonclassified protein) (Foran et al., 2017). Ud6_PL24 and Ud9_PL24 and the enzymes belonging to PL25 possessed lipobox. Although Ud2_PL24, Ud3_PL24, Ud4_PL24, Ud5_PL24, and Ud8_PL24 contained type I signal peptide, the remaining enzymes did not have any signal peptides. Although only one pectin lyase had a lipobox, many ulvan lyases possessed lipobox as well as alginate lyases, indicating that alginate and ulvan degradation activity of QB4 may be more effective than pectin degradation of the strain.

The unsaturated uronyl residue at the non-reducing end of the oligosaccharides produced by the ulvan lyases may be released by unsaturated glucuronyl hydrolases (GH105), because five putative unsaturated glucuronyl hydrolases, Ud15_GH105, Ud16_GH105, Ud17_GH105, Ud18_GH105, and Ud19_GH105 were found in the QB4 genome and showed similarity on unsaturated 3s-rhamnoglycuronyl hydrolase of Nonlabens ulvanivorans, which is known as a ulvan-degrading bacterium, based on BLASTp search (Table 3; Table S3) (Collén et al., 2014). After forming DKI by the enzymes, the following process may be the same as pectin utilization. In the ulvan-degrading process of $F$. agariphila, Rha3S-Xyl-Rha3S was also the main product by ulvan lyases (Reisky et al., 2019). First, Rha3S at the non-reducing end is desulfated by sulfatases. The amino acid sequences of two sulfatases, Ulu1 and Ulu2, in the QB4 genome showed high similarity (77.3 and 86.2\%) to the sulfatases, WP.032096151.1 and WP.632096147.1, located into ulvan utilization loci of Alteromonas sp. LOR (Foran et al., 2017), indicating that the two sulfatases may involve desulfation of the Rha3S. The Rha3 will remove the Rha3-Xyl-Rha3S by $\alpha$-L-rhamnosidase. Five $\alpha$-L-rhamnosidases classified into GH78 family, Ud20_GH78, Ud21_GH78, Ud22_GH78, Ud23_GH78, and Ud24_GH78, were found in the QB4 genome and showed similarity on rhamnosidase from Bacillus sp. GL1, Dictyoglomus thermophilum, Streptomyces avermitilis, and Bacteroides thetaiotaomicron (Table S3). Ud21_GH78, Ud22_GH78, Ud23_GH78, and Ud24_GH78 contained lipobox, suggesting that 
371 these enzymes are located on the cell surface. In addition, Ud20_GH78 and Ud22_GH78

372 possessed the CBM67 domain, which binds L-rhamnose in a calcium-dependent manner

373 (Fujimoto et al., 2013), suggesting that the two $\alpha$-L-rhamnosidases are the main components to

374 generate Rha3.

375 Rha3 metabolic pathway was described by Reisky et al., 2019. $\alpha$-L-rhamnose is converted to $\beta$ -

376 L-rhamnose by L-rhamnose mutarotase. Next, isomerization of the $\beta$-L-rhamnose to L-

377 rhamnulose is catalyzed by rhamnose isomerase. The product is converted to L-rhamnulose-1-

378 phosphate by pentulose/hexulose kinase (rhamnulokinase), and subsequently, the L-rhamnulose-

379 1-phosphate is cleaved into L-lactaldehyde and dihydroxyacetone phosphate by rhamnulose

380 aldolase. Finally, the L-lactaldehyde is converted to pyruvate by aldehyde dehydrogenase and

381 lactate dehydrogenase. Table 3 displayed that the corresponding genes (Ulu3, Ulu4, Ulu5, Ulu6,

382 Ulu7, and Ulu8) were found in the QB4 genome, and these proteins showed similarity on

383 corresponding proteins in the PDB database (Table S3). This result indicated that Rha3 is

384 metabolized by QB4 cells. The genes involving the metabolic pathway downstream of the

385 mutarotation in F. agariphila form a gene cluster (Reisky et al., 2019). However, the genes in

386 QB4 were randomly distributed in the genome.

387 Xyl will be released by $\beta$-xylosidase classified into GH3 and GH43 families. Based on the CAZy 388 database, seven GH43 (Ud25_GH43, Ud26_GH43, Ud27_GH43, Ud28_GH43, Ud29_GH43,

389 Ud30_GH43, and Ud31_GH43) proteins were found in the QB1 genome while F. agariphila

390 possessed two each of GH3 and GH43 proteins (Reisky et al., 2019). Ud25_GH43, Ud29_GH43,

391 and Ud31_GH43 were similar to glycoside hydrolases from Zobellia galactanivorans (50.00 \%)

392 and Halothermothrix orenii H 168 (45.75 and 39.10\%) (Table S3). The D-xylose is converted to

393 D-xylulose-5-phosphate via xylose isomerase and xylulose kinase, and then the product is passed

394 to the pentose phosphate pathway. Two genes encoding xylose isomerase (Ulu9) and xylulose

395 kinase (Ulu10) were found in the QB4 genome based on RAST server annotation and formed a

396 gene cluster with transcription repressor, $x y l R$ (Ulu11) (Table 3). These results suggested that

397 QB4 cells may use not only glucuronic acid but also L-rhamnose and D-xylose as carbon sources.

398 In addition, the presence of numerous genes encoding ulvan lyase may promote strong ulvan

399 degradation and its utilization. Actually, the QB4 cells exhibited a robust cell growth in the

400 presence of ulvan as well as that in the presence of alginate, as shown in Figure 1.

401 


\section{Genes for gellan gum degradation and utilization}

403 Gellan gum is depolymerized gellan lyases classified into PL33 family proteins. Gellan lyases 404 are found in several bacterial species, such as Bacillus sp. GL1, Geobacillus stearothermophilus 405 98, and Opitutaceae bacterium TAV5 (Hashimoto et al., 1997; Derekova et al., 2006; Helbert et 406 al., 2019). Gellan gum is degraded to tetrasaccharides composed of $[\rightarrow 3)-\beta$-D-glucose-( $1 \rightarrow 4)-\beta$ 407 D-glucuronic acid-( $1 \rightarrow 4)-\beta$-D-glucose- $(1 \rightarrow 4)-\alpha$-L-rhamnose- $(1 \rightarrow]$ by gellan lyases. The 408 tetrasaccharides are completely degraded to monosaccharides by $\beta$-D-glucosidase (GH1, GH2, 409 GH3, GH5, GH9, GH16, GH30, GH39, and GH116), unsaturated glucuronyl hydrolase (GH88 410 and GH105), and $\alpha$-L-rhamnosidase (GH78) (Hashimoto et al., 2003).

411 Figure 1 demonstrated that QB4 might also use gellan gum as a carbon source for its growth.

412 However, gellan lyases were not found in the genome. As shown in Table 3, QB4 possessed five 413 unsaturated glucuronyl hydrolases and five $\alpha$-L-rhamnosidases (Table 3). The hydrolysates, $414 \mathrm{DEH}$, and $\alpha$-L-rhamnose, may be metabolized by the pathway described above. In addition, one $415 \beta$-D-glucosidase classified into the GH1 family (Gd1-GH1) was also found in the genome, and 416 Gd1-GH1 showed high similarity (63.23 \%) on $\beta$-glucosidase A of Hungateiclostridium 417 thermocellum in the PDB database (Table 4; Table S3). $\beta$-D-glucose produced by the $\beta$-D418 glucosidases is converted to $\beta$-D-glucose-6-phosphate by glucokinase, and then the product is 419 converted to $\beta$-D-fructose-6-phosphate, which is an intermediate of glycolysis, by glucose-6420 phosphate isomerase. One of these genes, Gu1 and Gu2, were found in the QB4 genome (Table 421 4) and were similar on glucokinase and glucose-6-phosphate Isomerase of E. coli in PDB 422 database, respectively (Table S4), indicating that $\beta$-D-glucose is converted to $\beta$-D-fructose-6423 phosphate and is metabolized by glycolysis.

424

425 Discussion

426 Polysaccharides from plants, seaweed, and bacteria, especially their oligosaccharides, have 427 attracted considerable attention due to various biological activities. Thus, polysaccharide lyases 428 and glycosyl hydrolases were isolated from microorganisms, including fungi and bacteria.

429 The genus Catenovulum have been known as bacteria that can degrade agar and polysaccharide 430 containing uronic acids, such as alginate, pectin, and ulvan (Cui et al., 2014; Li et al., 2015; Lee 431 et al., 2019; Liu et al., 2019). Our previous report demonstrated that many genes encoding 432 polysaccharide degrading enzymes were present in the QB4 genome (Lau et al., 2019. In this 
433 study, expectedly, QB4 cells were able to degrade and used the polysaccharides as a carbon 434 source for their growth (Fig. 1). Especially, the QB4 cells exhibited a robust growth in the 435 presence of alginate and ulvan, suggesting that polysaccharides from marine algae are effectively 436 used by QB4 cells rather than that of plants and bacteria. The growth profile of QB4 was 437 different from $S$. degradans of which the cells reached the early stationary phase at $9 \mathrm{~h}$ with 438 alginate, glucose, and pectin (Takagi et al., 2016a).

439 Three pathways of pectin metabolisms were known in bacteria. One is the polygalacturonate 440 pathway metabolized unsaturated disaccharides. The pathway was found in bacteria, such as 441 Escherichia coli and phytopathogenic enterobacterium, Erwinia chrysanthemi (Chatterjee et al., 442 1985; Richard and Hilditch 2009). The other two pathways metabolize saturated galacturonate 443 through uronate isomerase (isomerase pathway) (Richard and Hilditch, 2009) or tagaturonate 444 epimerase (epimerase pathway) (Rodionova et al., 2012). Table 2 displayed that the QB4 445 possessed genes involved in the three pathways. However, QB4 cells did not grow in the 446 presence of saturated galacturonate (Fig. 2). Although a pectin-degrading marine bacterium, 447 Pseudoalteromonas sp. PS47, also has genes for the epimerase pathway, the strain was unable to 448 grow on saturated galacturonate (Hobbs et al., 2019). Hobbs and colleagues proposed that $449 \mathrm{GH} 28 \mathrm{~s}$ of the strain are periplasmic proteins and, thus, that saturated galacturonates would be 450 produced in the periplasm (Hobbs et al., 2019). It was suggested that the strain might be unable 451 to transport extracellular saturated galacturonate. On the other hand, although Pd11_GH28 452 containing Tat signal peptide would be exported into periplasm through Tat (for twin-arginine 453 translocation) system (Stanley et al., 2000), Pd12_GH28 would be secreted to the outside of the 454 cells via Sec system, suggesting that saturated galacturonate might be generated in the culture 455 broth in contrast to the strain PS47. However, the QB4 did not grow using saturated 456 galacturonate. Thus, it seems like the BQ4 cells are also unable to transport extracellular 457 saturated galacturonate as well as the strain PS47. In other cases, some investigators reported that 458 the isomerase pathway is not crucial for utilizing pectin in Dickeya dadantii (formerly $E$. 459 chrysanthemi) (Hugouvieux-Cotte-Pattat et al., 1996; Pédron et al., 2018). It was known that $D$. 460 dadantii belonging to the class Gammaproteobacteria, which is a plant pathogen and pectinolytic 461 bacterium, also possessed the polygalacturonate pathway and the isomerase pathway 462 (Hugouvieux-Cotte-Pattat et al., 1996). Even though the enzyme production of the isomerase 463 pathway was not impaired by a mutation in genes of the polygalacturonate pathway $(k d u D$ and 
$464 k d u I$ ), these mutants did not exhibit their growth on polygalacturonate (Hugouvieux-Cotte-Pattat 465 et al., 1996). In addition, the transcriptomic analysis of $D$. dadantii during the early colonization 466 on the plant leaf demonstrated that genes involved in the polygalacturonate pathway were 467 upregulated in the condition (Pédron et al., 2018). These results suggested that the isomerase 468 pathway was not the main pathway for utilizing pectin in the bacterium. The third pathway, 469 epimerase pathway, involving the conversion of D-tagatose to D-fructuronate by tagatose 470 epimerase (UxaE), was found in the hyperthermophilic bacterium, Thermotoga maritima, 471 belonging to the phylum Thermotoga. However, the pathway was not found in other bacteria, 472 such as Escherichia and Bacillus (Kuivanen et al., 2019). uxaE and other epimerase pathway 473 genes, such as hexuronate catabolism regulator ( $u x a R)$, fructuronate reductase (uxaD), predicted 474 D-mannonate utilization enzyme $(g n t E)$ constituted a regulon regulated by the GntR-like 475 transcription factor $\mathrm{UxaR}$ in the genus Thermotoga (Rodionova et al., 2012). A regulon is a gene 476 cluster or operon that is regulated by the same regulatory protein. However, Table 2 477 demonstrated that genes encoding Pu14, Pu15, and Pu16 were scattered in the chromosome and 478 plasmid, suggesting that $\mathrm{Pu} 14, \mathrm{Pu} 15$, and $\mathrm{Pu} 16$ might not constitute a regulon. In addition, figure 4792 demonstrated that the saturated galacturonate, which is a substrate of the epimerase pathway, 480 was not used by QB4 cells. These results suggested that the epimerase pathway might not 481 function in QB4.

482 Figure 1 demonstrated that the QB4 cells exhibited a robust growth using ulvan as a carbon 483 source. Qiao et al. (2020) fermented Catenovulum sp. LP using the shaking-flask method 484 containing $1.2 \%$ purified ulvan, of which the concentration was 6 times-higher than that of the 485 QB4. The bacterial culture reached the stationary phase at $36 \mathrm{~h}$ incubation period (Qiao et al., 486 2020) that was quite slower than that of the QB4. Table S5 showed that the QB4 genome 487 contained 14 ulvan lyase genes, which is the largest number compared to Siansivirga 488 zeaxanthinifaciens CC-SAMT-1 (8 genes) Tamlana sp. UJ94 (7 genes), Polaribacter sp. BM10 489 (7 genes), and Wenyingzhunangia fucanilytica CZ1127 (7 genes) in the CAZy database (Table 490 S5). The numerous genes in the QB4 genome may be crucial to efficiently degrade ulvan and to 491 stimulate robust growth.

492 Figure 1 demonstrated that the QB4 cells were capable of using gelzan as a carbon source, 493 however, no gallan lyases found in the QB4 genome. It was known that Bacillus sp. GL1 and G. 494 stearothermophilus 98, which possessed all genes for gellan gum degradation and utilization, 
495 were able to use gellan gum as a sole carbon source (Hashimoto et al., 1998; Derekova et al., 496 2006). On the other hand, although Paludisphaera borealis PX4 belonging to the order

497 Planctomycetales does not have any gellan lyases, the bacterium was capable of degrading gellan

498 gum (Ivanova et al., 2017). The authors suggested that an unsaturated glucuronyl hydrolase and 499 two $\alpha$-L-rhamnosidases of the bacterium are involving degrading gellan gum. Tables 3 and 4 500 showed that QB4 cells possess five unsaturated glucuronyl hydrolases, five $\alpha$-L-rhamnosidases, 501 and one $\beta$-D-glucosidase. We expected that these hydrolases play a critical role in degrading 502 gellan gum in QB4 cells. On the other hand, the lack of gellan lyases may cause lower 503 degradation efficiency. Thus, the cell growth with gellan gum was not robust compared to those 504 with other uronic acids.

505

506 Conclusion

507 The agar-degrading bacterium, Catenovulum sp. CCB-QB4, used uronic acids, including 508 alginate, pectin ulvan, and gellan gum as carbon sources for its growth. Especially, QB4 cells 509 exhibited a robust cell growth in the presence of alginate and ulvan from seaweed. In gene 510 analysis based on the CAZy database, a large number of polysaccharide lyases and hydrolases 511 involved in degrading these uronic acids were found in the QB4 genome. Many alginate lyases, 512 ulvan lyases, unsaturated glucuronyl hydrolases, $\alpha$-L-rhamnosidase, and $\beta$-xylosidase contained 513 lipobox, indicating that QB4 cells can effectively degrade alginate and ulvan and uptake the 514 oligosaccharides. Of course, genes for metabolizing the uronic acids were also present in the 515 QB4. These results were suggested that QB4 will become a source of novel uronic acid 516 degradation enzymes.

517

518

519

\section{Conflict of interest}

521 The authors do not have any conflict of interest in relation to the work described.

522

523

524

525 
526

527

528

529

530

531

532

533

534 Reference

535 Alves, A., Sousa, R.A., and Reis, R.L. 2013. A practical perspective on ulvan extracted from green 536 algae. J. Appl. Phycol. 25, 407-424.

537 Aparna, S., Parvathi, A., and Kaniyassery, A. 2020. Isolation and characterization of a moderately

538 halophilic Marinobacter phage-host system from the Arabian Sea. Envison. Monit. Assess. 192, 539199

540 Armenteros, J.J.A., Tsirigos, K.D., Sønderby, C.K., Petersen, T.N., Winther, O., Brunak, S., von

541 Heijine, G., and Nielsen, H. 2019. SignalP 5.0 improves signal peptide predictions using deep 542 neural networks. Nat. Biotechnol. 37, 420-423.

543 Bonnin, E., Garnier, C., and Ralet, M.-C. 2014. Pectin-modifying enzymes and pectin-derived 544 materials: applications and impacts. Appl. Microbiol. Biotechnol. 98, 519-532.

545 Chatterjee, A.K., Thurn, K.K., and Tyrell, D.J. 1985. Isolation and characterization of Tn5 546 insertion mutants of Erwinia chrysanthemi that are deficient in polygalacturonate catabolic 547 enzymes oligogalacturonate lyase and 3-deoxy-D-glycero-2,5-hexodiulosonate dehydrogenase. $J$. 548 Bacteriol. 162, 708-714.

549 Collén, P.N., Jeudy, A., Sassi, J.-F., Groisillier, A., Czjzek, M., Coutinho, P.M., and Helbert, W. 550 2014. A novel unsaturated $\beta$-glucuronyl hydrolase involved in ulvan degradation unveils the 551 versatility of stereochemistry requirements in family GH105. J. Biol. Chem. 289, 6199-6211.

552 Cui, F., Dong, S., Shi, X., Zhao, X., and Zhang, X.-H. 2014. Overexpression and characterization 553 of a novel thermostable $\beta$-agarase YM01-3, from marine bacterium Catenovulum agarivorans 554 YM01T. Mar. Drugs 12, 2731-2747.

555 de Lederkremer, R.M., and Marino, C. 2003. Acids and Other Products of Oxidation of Sugars. 556 Adv. Carbohydr. Chem. Biochem. 58, 199-306. 
557 Derekova, A., Sjøholm, C., Mandeva, R., Michailova, L., and Kambourova, M. 2006. Biosynthesis

558 of a thermostable gellan lyase by newly isolated and characterized strain of Geobacillus 559 stearothermophilus 98. Extremophiles 10, 321-326.

560 Dhillon, A., Sharma, K., Rajulapati, V., and Goyal, A. 2018. The multi-ligand binding first family

56135 Carbohydrate Binding Module (CBM35) of Clostridium thermocellum targets

562 rhamnogalacturonan I. Arch. Biochem. Biophys. 654, 194-208.

563 Dubey, A.K., Yadav, S., Kumar, M., Anand, G., and Yadav, D. 2016. Molecular biology of 564 microbial pectate lyase: a review. Br. Biotechnol. J. 13, 1-26.

565 Edwards, M.C., Henriksen, E.D., Yomano, L.P., Gardner, B.C., Sharma, L.N., Ingram, L.O., and

566 Peterson, J.D. 2011. Addition of genes for cellobiase and pectinolytic activity in Escherichia coli

567 for fuel ethanol production from pectin-rich lignocellulosic biomass. Appl. Environ. Microbiol. 568 77, 5184-5191.

569 Foran, E., Buravenkov, V., Kopel, M., Mizrahi, N., Shoshani, S., Helbert, W., and Banin, E. 2017.

570 Functional characterization of a novel "ulvan utilization loci" found in Alteromonas sp. LOR

571 genome. Algal Res. 25, 39-46.

572 Fujimoto, Z. 2013, Structure and function of carbohydrate-binding module families 13 and 42 of

573 glycoside hydrolases, comprising a $\beta$-trefoil fold. Biosci. Biotechnol. Bioch. 77, 1363-1371.

574 Furusawa, G., Lau, N.-S., Shu-Chien, A.C., Jaya-Ram, A., and Amirul, A.-A.A. 2015.

575 Identification of polyunsaturated fatty acid and diterpenoid biosynthesis pathways from draft 576 genome of Aureispira sp. CCB-QB1. Mar. Genomics 19, 39-44.

577 Hashimoto, W., Miyake, O., Nankai, H., and Murata, K. 2003. Molecular identification of an $\alpha$-L578 rhamnosidase from Bacillus sp. strain GL1 as an enzyme involved in complete metabolism of 579 gellan. Arch. Biochem Biophys. 415, 235-244.

580 Hashimoto, W., Miki, H., Nankai, H., Sato, N., Kawai, S., and Murata, K. 1998. Molecular Cloning 581 of Two Genes for $\beta$-d-Glucosidase inBacillussp. GL1 and Identification of One as a Gellan582 Degrading Enzyme. Arch. Biochem Biophys. 360, 1-9.

583 Hashimoto, W., Maesaka, K., Sato, N., Kimura, S., Yamamoto, K., Kumagai, H., and Murata, K. 584 1997. Microbial System for Polysaccharide Depolymerization: Enzymatic Route for Gellan 585 Depolymerization byBacillus sp. GL1. Arch. Biochem Biophys. 339: 17-23.

586 Helbert, W., Poulet, L., Drouillard, S., Mathieu, S., Loiodice, M., Couturier, M., Lombard, V., 587 Terrapon, N., Turchetto, J., Vincentelli, R., et al. 2019. Discovery of novel carbohydrate-active 
588 enzymes through the rational exploration of the protein sequences space. Proc. Natl. Acad. Sci. 589 USA. 116, 6063-6068.

590 Hien, N.Q., Nagasawa, N., Tham, L.X., Yoshii, F., Dang, V.H., Mitomo, H., Makuuchi, K., and 591 Kume, T. 2000. Growth-promotion of plants with depolymerized alginates by irradiation. Radiat. 592 Phys. Chem. 59, 97-101.

593 Hobbs, J.K., Hettle, A.G., Vickers, C., and Boraston, A.B. 2019. Biochemical reconstruction of a 594 metabolic pathway from a marine bacterium reveals its mechanism of pectin depolymerization. 595 Appl. Environ. Microbiol. 85, e02114-02118.

596 Hobbs, J.K., Lee, S.M., Robb, M., Hof, F., Barr, C., Abe, K.T., Hehemann, J.-H., McLean, R., 597 Abbott, W.A., and Boraston, A.B. 2016. KdgF, the missing link in the microbial metabolism of 598 uronate sugars from pectin and alginate. Proc. Natl. Acad. Sci. USA. 113, 6188-6193.

599 Hugouvieux-Cotte-Pattat, N., Condemine, G., and Shevchik, V.E. 2014. Bacterial pectate lyases, 600 structural and functional diversity. Environ. Microbiol. Rep. 6, 427-440.

601 Hutcheson, S.W., Zhang, H., and Suvorov, M. 2011. Carbohydrase systems of Saccharophagus 602 degradans degrading marine complex polysaccharides. Mar. Drugs 9, 645-665.

603 Iurciuc (Tincu), C.E., Lungu, C., Martin, P., and Popa, M. 2015, Gellan. pharmaceutical, medical 604 and cosmetic applications. Cellulose Chem. Technol. 51, 187-202.

605 Jansson, P.-E., Lindberg, B., and Sandford, P.A. 1983. Structural studies of gellan gum, an 606 extracellular polysaccharide elaborated by Pseudomonas elodea. Carbohydr. Res. 124, 135-139.

607 John, R.P., Anisha, G., Nampoothiri, K.M., and Pandey, A. 2011. Micro and macroalgal biomass: 608 a renewable source for bioethanol. Bioresour. Technol. 102, 186-193.

609 Juncker, A.S., Willenbrock, H., von Heijne, G., Brunak, S., Nielsen, H., and Krogh, A. 2003. 610 Prediction of lipoprotein signal peptides in Gram-negative bacteria. Protein Sci. 12, 1652-1662.

611 Kanehisa, M., and Goto, S. 2000. KEGG: kyoto encyclopedia of genes and genomes. Nucleic Acids 612 Res. 28, 27-30.

613 Kashyap, D.R., Vohra, P.K., Chopra, S., and Tewari, R. 2001. Applications of pectinases in the 614 commercial sector: a review. Bioresour. Technol. 77, 215-227.

615 Kawada, A., Hiura, N., Tajima, S., and Takahara, H. 1999. Alginate oligosaccharides stimulate 616 VEGF-mediated growth and migration of human endothelial cells. Arch. Dermatol. Res. 291, 542617547. 
618 Kawada, A., Hiura, N., Shiraiwa, M., Tajima, S., Hiruma, M., Hara, K., Ishibashi A., and Takahara,

619 H. 1997. Stimulation of human keratinocyte growth by alginate oligosaccharides, a possible 620 co-factor for epidermal growth factor in cell culture. FEBS lett. 408, 43-46.

621 Kim, D.H., Wang, D., Yun, E.J., Kim, S., Kim, S.R., and Kim, K.H. 2016. Validation of the 622 metabolic pathway of the alginate-derived monomer in Saccharophagus degradans 2-40T by gas 623 chromatography-mass spectrometry. Process Biochem. 51, 1374-1379.

624 Kim, S.-K., Thomas, N.V., and Li, X. 2011. Anticancer compounds from marine macroalgae and 625 their application as medicinal foods. Adv. Food Nutr. Res. 64, 213-24.

626 Kopel, M., Helbert, W., Belnik, Y., Buravenkov, V., Herman, A., and Banin, E. 2016. New family 627 of ulvan lyases identified in three isolates from the Alteromonadales order. J. Biol. Chem. 291, $628 \quad 5871-5878$.

629 Lau, N.-S., Tan, W.-R., Furusawa, G., and Amirul, A.A. 2019. Complete genome sequence of the 630 novel agarolytic Catenovulum-like strain CCB-QB4. Mar. Genomics 43, 50-53.

631 Lee, C.H., Lee, C.-R., and Hong, S.K. 2019. Biochemical characterization of a novel cold-adapted 632 agarotetraose-producing $\alpha$-agarase, AgaWS5, from Catenovulum sediminis WS1-A. Appl. 633 Microbiol. Biotechnol. 103, 8403-8411.

634 Li, Y., Huang, Z., Qiao, L., Gao, Y., Guan, H., Hwang, H., Aker, W.G., and Wang, P. 2015. 635 Purification and characterization of a novel enzyme produced by Catenovulum sp. LP and its 636 application in the pre-treatment to Ulva prolifera for bio-ethanol production. Process Biochem. $6375 \mathbf{5 0}, 799-806$.

638 Li, Q., Hu, F., Zhu, B., Ni, F., and Yao, Z. 2020. Insights into ulvan lyase: review of source, 639 biochemical characteristics, structure and catalytic mechanism. Crti. Rev. Biotechnol. 40, 432-441. 640 Liakos, I., Rizzello, L., Bayer, I.S., Pompa, P.P., Cingolani, R., and Athanassiou, A. 2013. 641 Controlled antiseptic release by alginate polymer films and beads. Carbohydr Polym. 92, 176-183. 642 Liu, J., Liu, Z., Jiang, C., and Mao, X. 2019. Biochemical Characterization and Substrate 643 Degradation Mode of a Novel $\alpha$-Agarase from Catenovulum agarivorans. J. Agric. Food Chem. 644 67, 10373-10379.

645 Liu J., Yang, S., Li, X., Yan, Q., Reaney, M.J.T., and Jiang, Z. 2019. Alginate oligosaccharides: 646 production, biological activities, and potential applications. Compr. Rev, Food Sci. F. 18, 18596471881. 
648 Mandalari, G., Palop, C.N., Tuohy, K., Gibson, G.R., Bennett, R.N., Waldron, K.W., Bisignano, 649 G., Narbad, A., and Faulds, C.B. 2007. In vitro evaluation of the prebiotic activity of a pectic 650 oligosaccharide-rich extract enzymatically derived from bergamot peel. Appl. Microbiol. 651 Biotechnol. 73, 1173-1179.

652 Martău, G.A., Mihai, M., and Vodnar, D.C. 2019. The use of chitosan, alginate, and pectin in the 653 biomedical and food sector-biocompatibility, bioadhesiveness, and biodegradability. Polymers 11, 654 1-28.

655 Meng, S., and Liu, Y. 2013. Alginate block fractions and their effects on membrane fouling. Water 656 Res. 47, 6618-6627.

657 Morelli. A. and Chiellini, F. 2010. Ulvan as a new type of biomaterial from renewable resources: 658 functionalization and hydrogel preparation. Macromol. Chem. Phys 211, 821-832.

659 Mudgil, D. 2017. The Interaction Between Insoluble and Soluble Fiber. In Samaan, R.A. (ed) 660 Dietary Fiber for the Prevention of Cardiovascular Disease, Academic Press, USA, pp. 35-59.

661 Munarin, F., Tanzi, M.C., and Petrini, P. 2012. Advances in biomedical applications of pectin gels. 662 Int. J. Biol. Macromol. 51, 681-689.

663 Pédron, J.P., Chapelle, E., Alunni, B., and van Gijsegem, F. 2018. Transcriptome analysis of the 664 Dikeya dadantii PecS regulon during the early stages of interaction with Arabidopsis thaliana. 665 Mol. Plant Pathol. 19, 647-663

666 Penhasi, A., and Meidan, V.M. 2015. Preparation and characterization of in situ ionic cross-linked 667 pectin films: unique biodegradable polymers. Carbohydr. Polym. 15, 254-260.

668 Pugsley, A.P., Chapon, C., and Schwartz, M. 1986. Extracellular pullulanase of Klebsiella 669 pneumoniae is a lipoprotein. J. Bacteriol. 166, 1083-1088.

670 Qiao, L., Yang, X., Xie, R., Du, C., Chi, Y., Zhang, J., and Wang, P. 2020. Efficient production of 671 ulvan lyase from Ulva prolifera by Catenovulum sp. LP based on stage-controlled fermentation 672 strategy. Algal Res. 46, 101812.

673 Qin, H.-M., Xu, P., Guo, Q., Cheng, X., Gao, D., Sun, D., Zhu, Z., and Li, F. 2018. Biochemical 674 characterization of a novel ulvan lyase from Pseudoalteromonas sp. strain PLSV. RSC Adv. 8, $6752610-2615$.

676 Qiao, L., Yang, X., Xie, R., Du, C., Chi, Y., Zhang, J., and Wang, P. 2020. Efficient production of 677 ulvan lyase from Ulva Prolifera by Catenovulum sp. LP based on stage-controlled fermentation 678 strategy. Algal Res. 46, 101812. 
679 Rahman, N., Dafader, N.C., and Banu, P. 2017. Preparation and Property Analysis of 680 Biodegradable Packaging Film from Alginate, Starch and Citric acid. J. Polym. Sci. Technol. 2, $68120-35$.

682 Reisky, L., Prechoux, A., Zühlke, M.-K., Bäumgen, M., Robb, C.S., Gerlach, N., Roret, T., 683 Stanetty, C., Larocque, R., Michel, G., et al., 2019. A marine bacterial enzymatic cascade degrades 684 the algal polysaccharide ulvan. Nat. Chem. Biol. 15, 803-812.

685 Richard, P., and Hilditch, S. 2009. D-galacturonic acid catabolism in microorganisms and its 686 biotechnological relevance. Appl. Microbiol. Biotechnol. 82, 597-604.

687 Rodionova, I., Scott, D.A., Grishin, N.V., Osterman, A.L., and Rodionov, D.A. 2012. 688 Tagaturonate-fructuronate epimerase UxaE, a novel enzyme in the hexuronate catabolic network 689 in Thermotoga maritima. Environ. Microbiol. 14, 2920-2934.

690 Stanley, N.R., Palmer, T., and Berks, B.C. 2000. The twin arginie consensus motif of Tat signal 691 peptides is involved in Sec-independent protein targeting in Escherichia coli. J. Biol. Chem. 275, 692 11591-11596.

693 Tabarsa, M., Han, J.H., Kim C.Y., and You, S.G. 2012. Molecular characteristics and 694 immunomodulatory activities of water-soluble sulfated polysaccharides from Ulva pertusa. J. 695 Med. Food 15, 135-144.

696 Takagi, T., Morisaka, H., Aburaya, S., Tatsukami, Y., Kuroda, K., and Ueda, M. 2016a. Putative 697 alginate assimilation process of the marine bacterium Saccharophagus degradans 2-40 based on 698 quantitative proteomic analysis. Mar. Biotechnol. 18, 15-23.

699 Takagi, T., Yokoi, T., Shibata, T., Morisaka, H., Kuroda, K., and Ueda, M. 2016b. Engineered 700 yeast whole-cell biocatalyst for direct degradation of alginate from macroalgae and production of 701 non-commercialized useful monosaccharide from alginate. Appl. Microbiol. Biotechnol. 100, $702 \quad 1723-1732$.

703 Takase, R., Ochiai, A., Mikami, B., Hashimoto, W., and Murata, K. 2010. Molecular identification 704 of unsaturated uronate reductase prerequisite for alginate metabolism in Sphingomonas sp. A1. 705 Biochim Biophys Acta. 1804, 1925-1936.

706 Takase, R., Mikami, B., Kawai, S., Murata, K., and Hashimoto, W. 2014. Structure-based 707 conversion of the coenzyme requirement of a short-chain dehydrogenase/reductase involved in 708 bacterial alginate metabolism. J. Biol. Chem. 289, 33198-33214. 
709 Takeda, H., Yoneyama, F., Kawai, S., Hashimoto, W., and Murata, K. 2011. Bioethanol

710 production from marine biomass alginate by metabolically engineered bacteria. Energy Environ.

711 Sci. 4, 2575-2581.

712 Thompson, J.D., Higgins, D.G., and Gibson, T.J. 1994. CLUSTAL W: improving the sensitivity

713 of progressive multiple sequence alignment through sequence weighting, position-specific gap

714 penalties and weight matrix choice. Nucleic Acids Res. 22, 4673-4680.

715 Tomoda, Y., Umemura, K., and Adachi, T. 1994. Promotion of barley root elongation under

716 hypoxic conditions by alginate lyase-lysate (ALL). Biosci. Biotechnol. Biochem. 58, 202-203.

717 Ulaganathan, T., Banin, E., Helbert, W., and Cygler, M. 2018a. Structural and functional

718 characterization of PL28 family ulvan lyase NLR48 from Nonlabens ulvanivorans. J. Biol. Chem.

719 293, 11564-11573.

720 Ulaganathan, T., Helbert, W., Kopel, M., Banin, E., and Cygler, M. 2018b. Structure-function

721 analyses of a PL24 family ulvan lyase reveal key features and suggest its catalytic mechanism. $J$.

722 Biol. Chem. 293, 4026-4036.

723 Valk, L.C., Luttik, M.A., de Ram, C., Pabst, M., van den Broek, M., van Loosdrecht, M.C.M., and

724 Pronk, J.T. 2020. A Novel D-Galacturonate Fermentation Pathway in Lactobacillus suebicus

725 Links Initial Reactions of the Galacturonate-Isomerase Route With the Phosphoketolase Pathway.

726 Front. Microbiol. 10, 3027.

727 Vendrusculo, C.T., Pereira, J.L., and Scamparini, A.R.P. 1994. Gellan gum: production and

728 properties. In Nishinari, K. and Doi, E. (eds) Food hydrocolloids: structure, properties and 729 function. Springer, Boston, MA, USA, pp. 91-95.

730 Venkatesan, J., Lowe, B., Anil, S., Manivasagan, P., Kheraif, A.A.A., Kang, K.-H., and Kim, S.-

731 K. 2015. Seaweed polysaccharides and their potential biomedical applications. Starch-Stärke 67, $732 \quad 381-390$.

733 Xie, W., Lin, B., Zhou, Z., Lu, G., Lun, J., Xia, C., Li, S., and Hu, Z. 2013. Characterization of a 734 novel $\beta$-agarase from an agar-degrading bacterium Catenovulum sp. X3. Appl. Microbiol. 735 Biotechnol. 97, 4907-4915.

736 Yin, Y., Mao, X., Yang, J., Chen, X., Mao, F., and Xu, Y. 2012. dbCAN: a web resource for 737 automated carbohydrate-active enzyme annotation. Nucleic Acids Res. 40, W445-W451. 
751

752

753

754

755

756

757

758 Figure legends

759 Figure 1. Growth confirmation of QB4 cultured with four different uronic acid-containing 760 polysaccharides, alginate (Alg), pectin (Pct), Ulvan (Ulv), and Gelzan (Gel). The growth was

761 measured by cfu/mL. Media with and without $0.2 \%$ glucose was used as positive (Glu) and

762 negative controls (NC), respectively. All data shown are mean values from three replicate

763 experiments. Error bars denote the standard deviation of triplicate samples.

764

765 Figure 2. Growth confirmation of QB4 cultured with saturated galacturonate (Sat. Gal). The 766 growth was measured as optical density $\left(\mathrm{OD} 600_{\mathrm{nm}}\right)$. Media with and without $0.2 \%$ glucose was 767 used as positive (Glu) and negative controls (NC), respectively. All data shown are mean values 768 from three replicate experiments. Error bars denote the standard deviation of triplicate samples. 769 
Figure 1

Growth confirmation of QB4 cultured with four different uronic acid-containing polysaccharides, alginate (Alg), pectin (Pct), Ulvan (Ulv), and Gelzan (Gel).

The growth was measured by $\mathrm{cfu} / \mathrm{mL}$. Media with and without $0.2 \%$ glucose was used as positive (Glu) and negative controls (NC), respectively. All data shown are mean values from three replicate experiments. Error bars denote the standard deviation of triplicate samples.

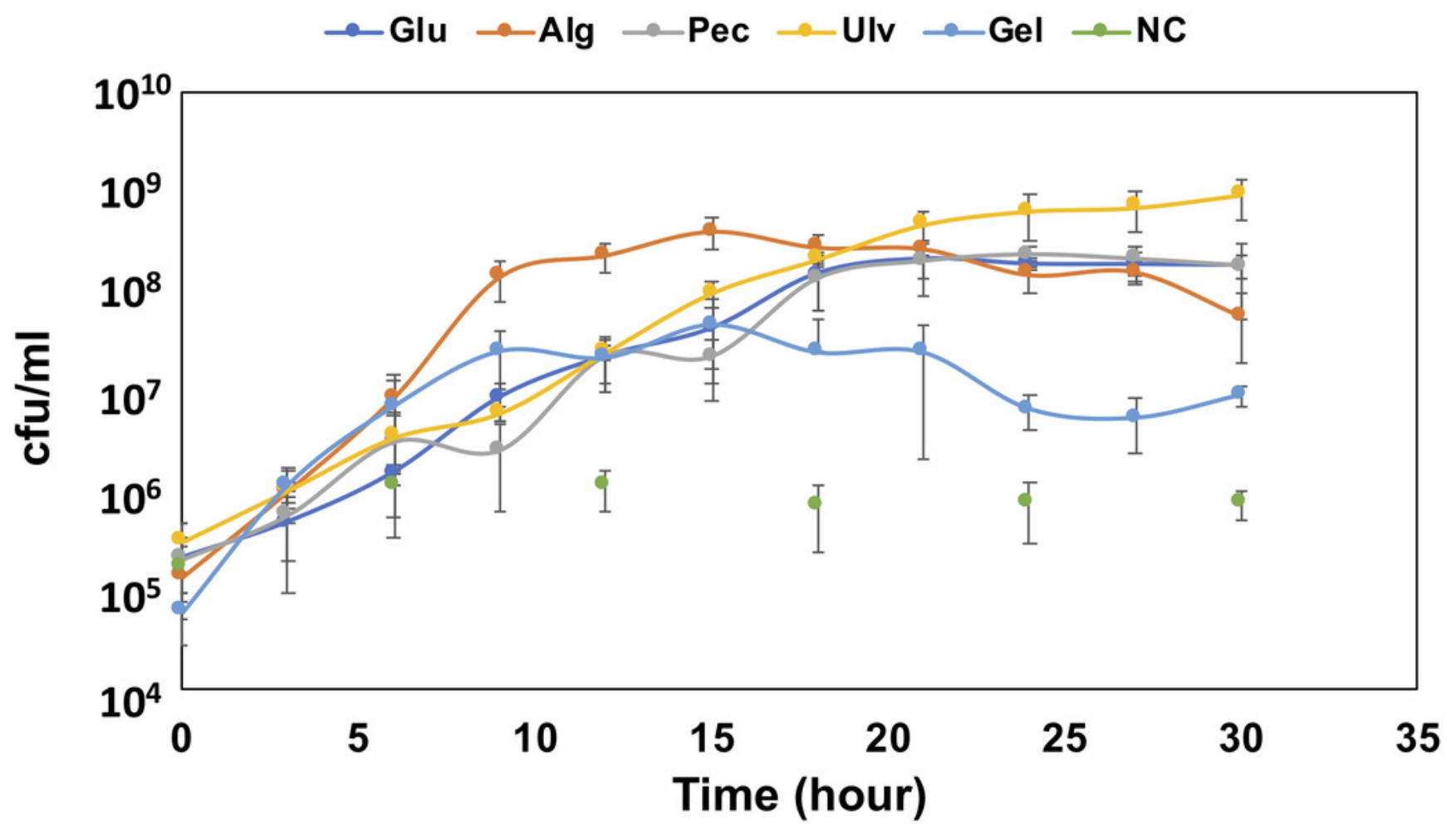


Figure 2

Growth confirmation of QB4 cultured with saturated galacturonate (Sat. Gal).

The growth was measured as optical density $\left(0 \mathrm{OD} 600_{\mathrm{nm}}\right)$. Media with and without $0.2 \%$ glucose was used as positive (Glu) and negative controls (NC), respectively. All data shown are mean values from three replicate experiments. Error bars denote the standard deviation of triplicate samples.

$\multimap$ Glu $\rightarrow$ Gal. mono. $\multimap$ NC

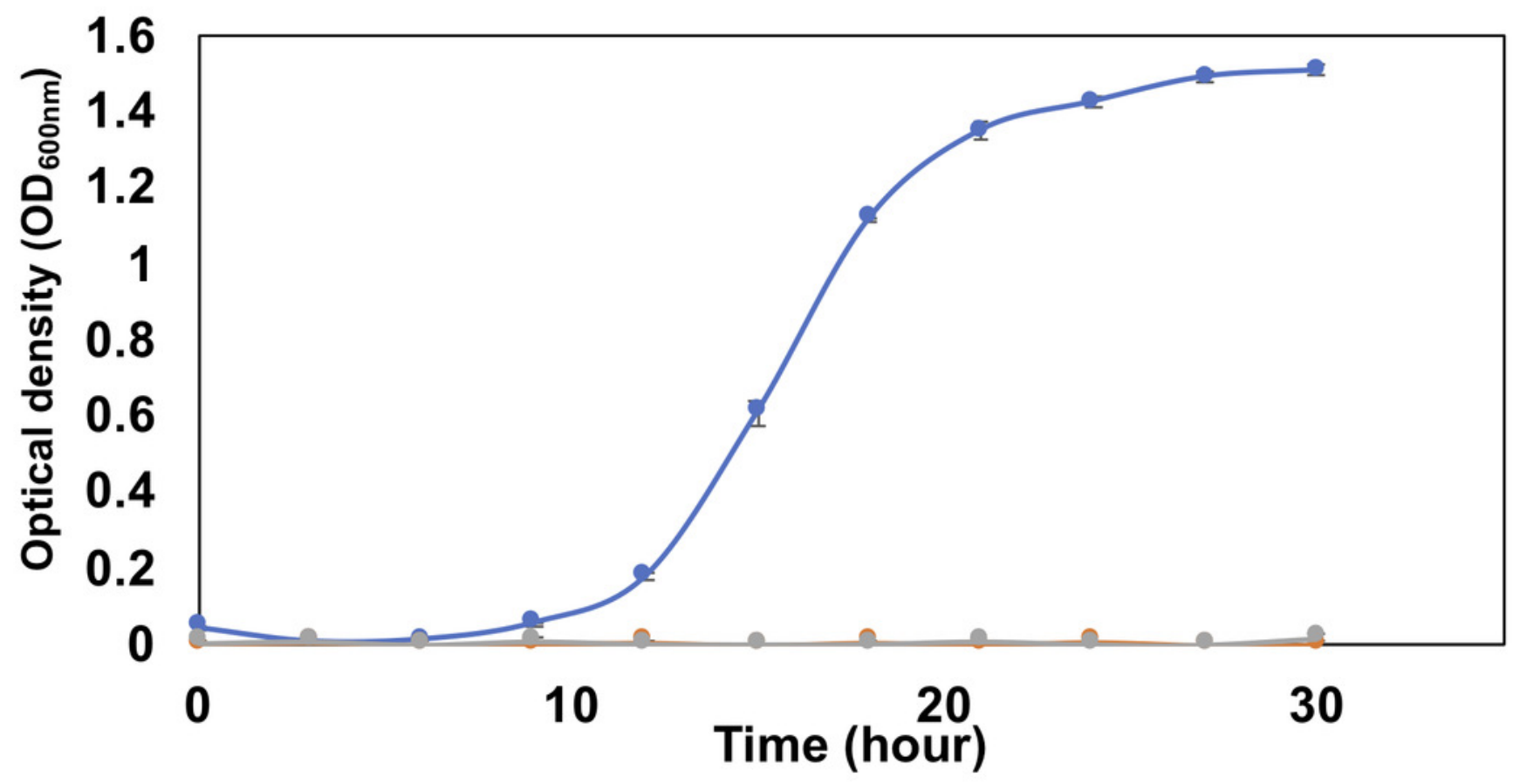




\section{Table 1 (on next page)}

Genes involving in alginate metabolism. 
1 Table 1. Genes involving in alginate metabolism.

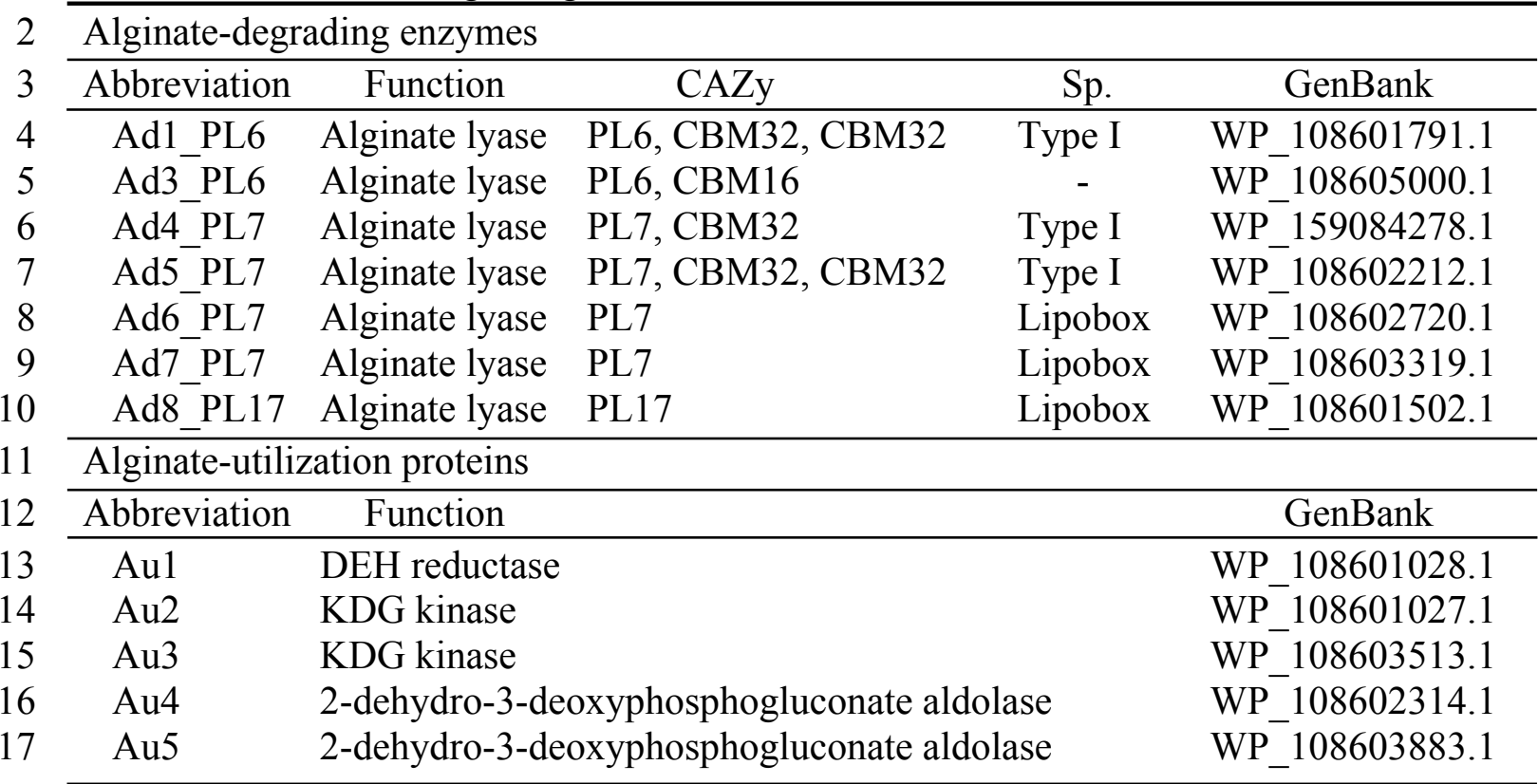

18 Sp. indicates signal peptide.

19

20

21 
Table 2 (on next page)

Genes involving in pectin metabolism. 
Table 2. Genes involving in pectin metabolism.

\begin{tabular}{|c|c|c|c|c|}
\hline \multicolumn{5}{|c|}{ Pectin-degrading enzymes } \\
\hline Abbreviation & Function & CAZy & Sp. & GenBank \\
\hline Pd1_PL1 & Pectate Lyase (Plasmid) & PL1, CBM13 & Type I & WP_108605188.1 \\
\hline Pd2_PL1 & Pectate Lyase (Plasmid) & PL1, CBM35, CBM77 & - & WP_108605291.1 \\
\hline Pd3_PL1 & Pectate Lyase (Plasmid) & PL1, CBM35 & Type I & AWB69198 \\
\hline Pd4_PL1 & Pectate Lyase (Plasmid) & PL1, CE8 & Type I & WP_159084287.1 \\
\hline Pd5_PL1 & Pectate Lyase (Plasmid) & PL1, CE8 & Lipobox & WP_108605272.1 \\
\hline Pd6_PL3 & Pectate Lyase (Plasmid) & PL3, CBM13 & Lipobox & WP_108605187.1 \\
\hline Pd7_PL10 & Pectate Lyase (Plasmid) & PL10 & Type I & WP_108605295.1 \\
\hline Pd8_GH105 & Unsaturated galacturonyl hydrolases (Plasmid) & GH105 & Lipobox & WP_108605228.1 \\
\hline $\mathrm{Pd} 9{ }^{-} \mathrm{GH} 105$ & Unsaturated galacturonyl hydrolases (Plasmid) & GH105 & Type I & WP- 108605230.1 \\
\hline Pd10_GH105 & Unsaturated galacturonyl hydrolases (Plasmid) & GH105 & Lipobox & WP_108605292.1 \\
\hline Pd11_GH28 & Polygalacturonases (Plasmid) & GH28 & Tat & WP_108605238.1 \\
\hline Pd12_GH28 & Polygalacturonases (Plasmid) & GH28 & Type I & WP_108605277.1 \\
\hline \multicolumn{5}{|c|}{ Pectin-utilization proteins } \\
\hline Abbreviation & Function & & & GenBank \\
\hline $\mathrm{Pul}$ & \multicolumn{3}{|l|}{ 5-dehydro-4-deoxy-D-glucuronate isomerase (KduI) } & WP_108601704.1 \\
\hline $\mathrm{Pu} 2$ & \multicolumn{3}{|l|}{ 5-dehydro-4-deoxy-D-glucuronate isomerase (KduI) } & WP_108601929.1 \\
\hline Pu3 & \multicolumn{3}{|l|}{ 5-dehydro-4-deoxy-D-glucuronate isomerase (KduI) } & WP_108602166.1 \\
\hline $\mathrm{Pu} 4$ & \multicolumn{3}{|c|}{ 5-dehydro-4-deoxy-D-glucuronate isomerase (KduI) (Plasmid) } & WP_108605242.1 \\
\hline Pu5 & \multicolumn{3}{|c|}{ 2-dehydro-3-deoxy-D-gluconate 5-dehydrogenase (KduD) } & WP- 108601158.1 \\
\hline Pu6 & \multicolumn{3}{|c|}{ 2-dehydro-3-deoxy-D-gluconate 5-dehydrogenase (KduD) } & WP_108601705.1 \\
\hline $\mathrm{Pu} 7$ & \multicolumn{3}{|c|}{ 2-dehydro-3-deoxy-D-gluconate 5-dehydrogenase (KduD) } & WP_108602165.1 \\
\hline Pu8 & \multicolumn{3}{|c|}{ 2-dehydro-3-deoxy-D-gluconate 5-dehydrogenase (KduD) (Plasmid) } & WP_108605243.1 \\
\hline Pu9 & \multicolumn{3}{|c|}{ Glucuronate isomerase } & WP_108601474.1 \\
\hline Pu10 & \multicolumn{3}{|l|}{ Glucuronate isomerase } & WP_108604964.1 \\
\hline Pu11 & \multicolumn{3}{|l|}{ Glucuronate isomerase (Plasmid) } & WP_108605248.1 \\
\hline Pu12 & \multicolumn{3}{|l|}{ Tagaturonate reductase } & WP_108601943.1 \\
\hline Pu13 & \multicolumn{3}{|l|}{ Altronate hydrolase } & WP_108601944.1 \\
\hline Pu14 & \multicolumn{3}{|l|}{ Tagaturonate epimerase (Plasmid) } & WP_108605252.1 \\
\hline Pu15 & \multicolumn{3}{|l|}{ Fructuronate reductase } & WP_108602164.1 \\
\hline Pu16 & \multicolumn{3}{|l|}{ Mannoate dehydratese } & WP_108601469.1 \\
\hline
\end{tabular}




\section{PeerJ}

34 Plasmid indicated that the gene is present in plasmid, not the genome. 
Table 3 (on next page)

Genes involving in ulvan metabolism. 
Table 3. Genes involving in ulvan metabolism.

\begin{tabular}{|c|c|c|c|c|}
\hline \multicolumn{5}{|c|}{ Ulvan-degrading enzymes } \\
\hline Abbreviation & Function & CAZy & GenBank & \\
\hline Ud1_PL24 & Ulvan lyase & PL24 & & WP_108601530.1 \\
\hline $\mathrm{Ud} 2 \_\mathrm{PL} 24$ & Ulvan lyase & PL24 & Type I & WP_108601531.1 \\
\hline Ud3_PL24 & Ulvan lyase & PL24 & Type I & WP_108604943.1 \\
\hline Ud4_PL24 & Ulvan lyase & PL24 & Type I & WP_108601549.1 \\
\hline Ud5_PL24 & Ulvan lyase & PL24 & Type I & WP_108601554.1 \\
\hline Ud6_PL24 & Ulvan lyase & PL24 & Lipobox & WP_108602230.1 \\
\hline Ud7_PL24 & Ulvan lyase & PL24, CBM32 & & WP_108604992.1 \\
\hline Ud8_PL24 & Ulvan lyase & PL24 & Type I & WP_108602276.1 \\
\hline Ud9_PL24 & Ulvan lyase & PL24 & Lipobox & WP_108602290.1 \\
\hline Ud10_PL25 & Ulvan lyase & PL25 & Lipobox & WP_108601547.1 \\
\hline Ud11-PL25 & Ulvan lyase & PL25 & Lipobox & WP-108601636.1 \\
\hline Ud12_PL25 & Ulvan lyase & PL25 & Lipobox & WP_108601685.1 \\
\hline Ud13_PL25 & Ulvan lyase & PL25 & Lipobox & $\mathrm{WP}_{-}^{-} 108602265.1$ \\
\hline Ud14_PL40 & Ulvan lyase & PL40 & & WP_108602237.1 \\
\hline Ud15-GH105 & Unsaturated glucuronyl hydrolase & GH105 & Lipobox & $\mathrm{WP}^{-} 108601620.1$ \\
\hline Ud16_GH105 & Unsaturated glucuronyl hydrolase & GH105 & Lipobox & WP_108601696.1 \\
\hline Ud17_GH105 & Unsaturated glucuronyl hydrolase & GH105 & Lipobox & WP_108601928.1 \\
\hline Ud18_GH105 & Unsaturated glucuronyl hydrolase & GH105 & Lipobox & WP_108602225.1 \\
\hline Ud19_GH105 & Unsaturated glucuronyl hydrolase & GH105 & Lipobox & WP_108602277.1 \\
\hline Ud20_GH78 & $\alpha$-L-rhamnosidase & GH78, CBM67 & Type I & WP_108601540.1 \\
\hline Ud21-GH78 & $\alpha$-L-rhamnosidase & GH78 & Lipobox & WP-108604942.1 \\
\hline Ud22_GH78 & $\alpha$-L-rhamnosidase & GH78, CBM67 & Lipobox & WP_108601635.1 \\
\hline Ud23_GH78 & $\alpha$-L-rhamnosidase & GH78 & Lipobox & WP_108602268.1 \\
\hline Ud24_GH78 & $\alpha$-L-rhamnosidase & GH78 & Lipobox & WP_108602275.1 \\
\hline Ud25_GH43 & Putative $\beta$-xylosidase & GH43 & Type I & $\mathrm{WP}_{-}^{-} 108601365.1$ \\
\hline Ud26_GH43 & Putative $\beta$-xylosidase & GH43 & Lipobox & WP_108601561.1 \\
\hline $\mathrm{Ud} 27-\mathrm{GH} 43$ & $\beta$-xylosidase & GH43 & Type I & WP_108601631.1 \\
\hline Ud28_GH43 & $\beta$-xylosidase & GH43 & Lipobox & WP_108602133.1 \\
\hline Ud29_GH43 & $\beta$-xylosidase & GH43 & Lipobox & WP_159084088.1 \\
\hline Ud30_GH43 & $\beta$-xylosidase & GH43 & Lipobox & WP_108602377.1 \\
\hline
\end{tabular}




\begin{tabular}{|c|c|c|c|}
\hline 34 & Ud31_GH43 & $\beta$-xylosidase & WP_108602397.1 \\
\hline 35 & \multicolumn{3}{|c|}{ Ulvan-utilization proteins } \\
\hline 36 & Abbreviation & Function & GenBank \\
\hline 37 & Ulu1 & Sulfatase & WP_108601682.1 \\
\hline 38 & Ulu2 & Sulfatase & WP_108601697.1 \\
\hline 39 & Ulu3 & L-rhamnose mutarotase & WP_108601622.1 \\
\hline 40 & Ulu4 & Rhamnose isomerase & WP_108601699.1 \\
\hline 41 & Ulu5 & Pentulose/hexulose kinase (rhamnulokinase) & WP_108602148.1 \\
\hline 42 & Ulu6 & Rhamnulose aldolase & WP_108604932.1 \\
\hline 43 & Ulu7 & Aldehyde dehydrogenase & WP_108601159.1 \\
\hline 44 & Ulu8 & Lactate dehydrogenase & WP_108602249.1 \\
\hline 45 & Ulu9 & Xylose isomerase & WP_108604489.1 \\
\hline 46 & Ulu10 & Xylulose kinase & WP_108604490.1 \\
\hline 47 & Ulu11 & Transcriptional regulatory protein XylR & WP_10860449.1 \\
\hline
\end{tabular}


Table 4 (on next page)

Genes involving in ulvan metabolism. 
1 Table 3. Genes involving in ulvan metabolism.

2 Gellan-degrading enzymes

3 Abbreviation Function

CAZy Sp.

GenBank

4 Gd1_GH1 $\beta$-D-glucosidase

$\mathrm{GH} 1$

WP_159084202.1

5 Gellan-utilization proteins

6 Abbreviation Function

GenBank

7 Gul Glucokinase WP_108603884.1

8 Gu2 Glucose-6-phosphate isomerase WP_108604264.1

9 